\title{
Energy loss at zero temperature from extremal black holes
}

\author{
Moslem Ahmadvand $^{\mathrm{a}}$, Kazem Bitaghsir Fadafan ${ }^{\mathrm{b}}$ \\ Faculty of Physics, Shahrood University of Technology, P.O. Box 3619995161, Shahrood, Iran
}

Received: 8 February 2016 / Accepted: 2 October 2018 / Published online: 11 October 2018

(c) The Author(s) 2018

\begin{abstract}
Using the AdS/CFT correspondence, we probe the extremal black holes by studying the energy loss of a moving heavy point particle in a strongly-coupled boundary field theory at zero temperature and finite charge density. We first consider the extremal AdS-Reissner-Nordström (AdSRN) background black hole in the bulk and find an analytic solution of drag force which depends on the finite charge density of the boundary field theory. By studying the near horizon geometry of trailing string in the bulk, one finds the IR divergency does not show the logarithmic behavior. This result would be a general property of extremal backgrounds. We also extend our study to the extremal backgrounds with vanishing entropy density in appropriate extremal limits. After studying the Langevin coefficients, it is shown that in the extremal black hole backgrounds the longitudinal Langevin diffusion coefficient along the semiclassical string motion is larger compared to that of the transverse direction.
\end{abstract}

\section{Introduction}

Strongly-coupled systems describe interesting phenomena in different areas of physics. Study of such systems is theoretically difficult, then one should develop alternative theoretical tools to understand them better. In the framework of gauge/gravity correspondence, such systems with a large number of degrees of freedom could be realized holographically as classical gravity in a higher dimension [1-4]. Besides, from this correspondence to study the field theory states at nonzero temperature and density, one should consider geometries with a black brane horizon [5]. The equilibrium thermodynamic properties of such states are described by black hole thermodynamics [6].

In this paper, we consider zero temperature and finite density systems which correspond to extremal black branes.

\footnotetext{
a e-mail: ahmadvand@ shahroodut.ac.ir

be-mail: bitaghsir@shahroodut.ac.ir
}

They have vanishing surface gravity and Hawking temperature. Also, given a set of charges, they have the lowest possible mass. Therefore, they can be thought of as ground states of the nonextremal black holes and one finds that all supersymmetric BPS black holes are necessarily extremal in supersymmetric gravity theories. Because of the relation between horizon area and entropy, such extremal black branes are dual to field theory states with zero temperature and a large nonzero entropy density. This behavior is non-trivial and somewhat problematic to understand because, from the third law of thermodynamics, one expects zero entropy at zero temperature. Based on these reasons, extremal black holes have been extensively studied, for example in the case of quantum properties of black holes and microstate counting. Another example of such applications is studying the quantum critical phenomena in condensed matter systems [7]. The authors studied the geometry of extremal Anti-de Sitter Reissner-Nordstrom (AdSRN) in $d+1$ black brane where the dual field theory is supposed to be a $d$-dimensional stronglycoupled system at zero temperature and finite $U(1)$ charge density. To probe this system, they studied scalars as well as spinors. Some transport coefficients such as conductivity and viscosity have been studied using $A d S_{4} R N$. It was shown that the ratio of shear viscosity $(\eta)$ to the entropy density $(s)$ is $\frac{1}{4 \pi}[8-11]$. As a result, one may generalize some universal arguments at the finite temperature to field theory at zero temperature using extremal backgrounds.

The extremal backgrounds with vanishing entropy density in appropriate extremal limits are an interesting research area. Extremal black holes with a vanishing one-cycle on the horizon and with the vanishing horizon area have been studied in [12]. The different aspects of a given Extremal Vanishing Horizon $(\mathrm{EVH})$ black hole, its near-horizon geometry and a possible dual two-dimensional conformal field theory (CFT) picture for the corresponding excitations have been studied in $[13,14]$. They also discuss the EVH in various theories in diverse dimensions and EVH/CFT proposal. Moreover, dynamical laws of near horizon extremal geometries 
were discussed in $[15,16]$. Using "top-down" construction, the four and five-dimensional extremal black holes have been studied in [17-19]. They consider extremal black branes with a singular horizon and zero ground state entropy which is dual to zero temperature $\mathcal{N}=4$ Super-Yang-Mills theory with two equal nonvanishing chemical potentials. The singularities are related to divergences of the scalars as well as of curvature invariances and they can be avoided by turning on small but non-zero horizon radius $\left(r_{h}\right)$. One should notice that in the infrared region (IR) of such geometries there is no $A d S_{2}$ region which is interesting because in the extremal limit of more general black holes like AdSRN the $A d S_{2}$ regions are responsible for many universal properties of the boundary field theory at zero temperature [20].

To study field theory properties of such systems, one may use the linear response of the theory to fermionic operators as in [17]. Then Fermi surface singularities and the spectrum of fluctuations should be found and finally classified different behaviors as Fermi liquid, non-Fermi liquid, or marginal Fermi liquid. Results can be related to the experimental observations like strange metals in cuprates and in heavy fermion systems [21].

In this paper, we probe the extremal black holes by studying energy loss of a moving heavy point particle. In the case of quark-gluon-plasma (QGP) produced at RHIC and LHC, the energy loss of heavy probes is an important subject [22]. Studying such phenomena at strong coupling needs nonperturbative and time-dependent methods which are inadequate for describing [23,24]. Using gauge/gravity duality, the energy loss of heavy quarks was initially investigated in $[25,26]$. They considered a heavy probe quark traveling with constant linear velocity through the strongly coupled plasma and found the energy required to keep it in uniform motion. One finds that the energy loss is proportional to the momentum of the quark and concludes that the energy loss mechanism is such as a drag force.

Study of the energy loss of heavy probes at zero temperature has been done in the case of strongly-coupled superfluids in [27]. They use gauge/gravity correspondence and model a superfluid to study its response to a heavy pointlike probe. It was shown that there is a critical velocity which was determined from the bulk geometry. They also study the stochastic force acting on the moving heavy probe and found the transverse and longitudinal mean momentum transfer. The extension of the idea of using AdS/CFT to study energy loss of extended defects in strongly-coupled systems has been done in [28]. They consider bulk gravities created by general $D p$ branes as well as holographic superfluids. For the superfluid case, it was confirmed that there is a cutoff velocity, below which the probe experiences no drag force.

In this paper, we consider extremal black holes to study the drag force on the heavy probe. We find an analytic solution which depends on the chemical potential, quadratically.
Increasing the charge density leads to increasing of the drag force at a constant velocity. Decelerating of the probe at zero temperature can be studied by considering the behavior of trailing string after small perturbations. Interestingly, we find that the IR divergency does not show the logarithmic behavior, while it is a generic feature at finite temperature case. As a result, by studying the shape of trailing string in the near horizon geometry of extremal background, one concludes that the IR divergency of extremal black hole would not be the logarithmic case.

We also study Brownian motion of the probe and calculate the transverse $\left(\kappa_{T}\right)$ and longitudinal $\left(\kappa_{L}\right)$ mean momentum transfer at zero temperature and finite density. Such stochastic motion provides important observables in QGP, where related physics can be found in [33]. It has been shown that in generic isotropic holographic theories $\kappa_{L} / \kappa_{T}>1$. This is a universal relation and a way to violate is to consider anisotropic geometries [35]. We investigate that the universal behavior also exist in holographic extremal backgrounds.

The Langevin coefficients are defined in terms of the world-sheet temperature of trailing string $T_{w s}$. We show that $T_{w s}$ in the extremal backgrounds is finite while the boundary field theory temperature $T$ is zero. Since the system is out of equilibrium, $T_{w s}$ is different from the boundary temperature. For non-extremal backgrounds, it was argued that $T_{w s}$ can be lower than that of the heat bath $T$, then the non-equilibrium noise can be smaller than that in equilibrium case [34]. While this is not the case for the extremal backgrounds where the boundary field theory temperature is zero and always $T_{w s}$ is greater than $T=0$. The generic conditions for a negative excess noise have been discussed in [35].

Curiously, we extend our study to extremal backgrounds with vanishing entropy density in appropriate extremal limits. We first consider 5-dimensional extremal 3-charge black holes denoted as $(2+1) \mathrm{QBH}$ when two of charges are equal. In this case, there are two different extremal limits for vanishing temperature and entropy. Interestingly, we find an analytic result for the drag force and it is shown that it does not change in these limits. We also compute $\kappa_{L} / \kappa_{T}$ in this extremal case and it is found that it obeys the universal property [35].

This paper is organized as follows. In the next section, we will consider AdSRN extremal geometry in five dimensions and study the drag force at zero temperature. We also study in this section the stochastic behavior of the point particle. We extend our results to the case of $\mathcal{N}=4$ SYM theory with a 3-charge black hole (3QBH) in Sect. 3. In Sect. 4, we consider 10 dimensional 3QBH and study the same physics. In the last section, we summarize our results. 


\section{Energy loss from extremal AdSRN}

In this section, we study the drag force on a moving heavy probe in the gauge theory at zero temperature and finite density. From the AdS/CFT correspondence, the probe is the end of the string which hangs from boundary to the AdSRN background. The RN black hole with negative cosmological constant is given by the following action [7]:

$S=-\frac{1}{2 \kappa^{2}} \int d^{5} x \sqrt{-g}\left(R+\frac{12}{L^{2}}-\frac{L^{2}}{g_{F}^{2}} F_{\mu \nu} F^{\mu \nu}\right)$,

here $\kappa^{2}=8 \pi G, L$ is AdS radius, $g_{F}$ is an effective dimensionless gauge coupling constant, and $F_{\mu \nu}=\partial_{\mu} A_{\nu}-\partial_{\nu} A_{\mu}$ where $A_{\mu}$ is $U(1)$ gauge field. The 5-dimentional solution of equation of motion coming from this action can be obtained as $A d S_{5} \mathrm{RN}$ black hole

$d s^{2}=\frac{r^{2}}{L^{2}}\left(-f d t^{2}+d \vec{x}^{2}\right)+\frac{L^{2}}{r^{2} f} d r^{2}$,

here

$f=1-\frac{m}{r^{4}}+\frac{q^{2}}{r^{6}}, \quad A_{t}=\mu\left(1-\frac{r_{h}^{2}}{r^{2}}\right)$.

and $t, \vec{x}$ are time and space coordinates in the boundary and $r$ denotes the radial direction. We consider the event horizon as $r_{h}$ where $f\left(r_{h}\right)=0$, then one can rewrite $f(r)$ as follows:

$f=1-\frac{r_{h}^{4}}{r^{4}}+\frac{q^{2}}{r^{6} r_{h}^{2}}\left(r_{h}^{2}-r^{2}\right)$.

The black hole mass $m$ and the charge $q$ in terms of $r_{h}$ are given as

$m=r_{h}^{4}+\frac{q^{2}}{r_{h}^{2}}, \quad q^{2}=\mu^{2} n^{2} r_{h}^{4}$,

here $n^{2}=4 L^{4} / 3 g_{F}^{2}$. The Hawking temperature and entropy of black hole are given by

$T=\frac{r_{h}}{\pi L^{2}}\left(1-\frac{q^{2}}{2 r_{h}^{6}}\right), \quad S=\frac{1}{4 G}\left(\frac{r_{h}}{L}\right)^{3}$.

In the extremal limit, $q^{2}=2 r_{h}^{6}$ and the temperature vanishes. As is clear in this limit, the Hawking temperature vanishes while the horizon area is finite. This is one of the peculiar properties of the AdSRN black holes. It would be interpreted as a finite ground state degeneracy which is unusual in the dual field theory. We focus on these systems and study the drag force.

\subsection{Finding drag force from holography}

In the boundary field theory side, an external heavy probe can be described by a classical string that has a single end point at the boundary and extends down to the horizon. Because of the nonzero finite density, one expects a nonzero drag force. The trailing string dynamics is captured by the Nambu-Goto action as follows:

$S=-\frac{1}{2 \pi \alpha^{\prime}} \int d \sigma d \tau \sqrt{-\operatorname{det} g_{a b}}$.

here $g_{a b}$ is the induced metric on the string world-sheet. The $(\sigma, \tau)$ coordinates parameterize $g_{a b}$ where $a, b$ run over these two dimensions. By choosing $(\tau=t, \sigma=r)$ and defining $\dot{X}=\partial_{\tau} X, X^{\prime}=\partial_{\sigma} X$ and $\dot{X} \cdot X^{\prime}=\dot{X}^{\mu} X^{\prime \nu} G_{\mu \nu}$, where $G_{\mu \nu}$ is the background metric (2), one finds

$$
\text { - det } g=\left(\dot{X} \cdot X^{\prime}\right)^{2}-X^{\prime 2} \dot{X}^{2}=1-\frac{v^{2}}{f}+\frac{r^{4}}{L^{4}} x^{\prime 2} f \text {. }
$$

The string in this case trails behind its boundary endpoint as it moves at constant velocity in the $x$ direction

$x(r, t)=v t+x(r), \quad x^{2}=x^{3}=0$.

The equation of motion leads to

$x^{\prime 2}=\frac{L^{4} C^{2}\left(1-v^{2} f^{-1}\right)}{r^{4} f\left(r^{4} f L^{-4}-C^{2}\right)}$,

here $C$ is a constant of motion. To have a hanging string from boundary to the horizon, $x^{\prime 2}$ should be positive everywhere, i.e. both numerator and denominator of (10) should change sign at the same point. This critical point is denoted as $r_{c}$ and one finds the constant of motion in terms of it

$C=v r_{c}^{2} L^{-2}$.

The drag force experienced by the heavy probe is calculated by the following equation:

$F=-\frac{1}{2 \pi \alpha^{\prime}} \frac{\partial \mathcal{L}}{\partial x^{\prime}}=-\frac{1}{2 \pi \alpha^{\prime}} \frac{v r_{c}^{2}}{L^{2}}$.

By changing variables as $\beta^{-2}=1-v^{2}, y=r_{c}^{2}$, we try to find $r_{c}$ by solving $f\left(r_{c}\right)-v^{2}=0$. One finds this equation in terms of the new variables in non-extremal case as follows:

$\tilde{y}^{3}-\beta^{2}\left(r_{h}^{4}+\frac{q^{2}}{r_{h}^{2}}\right) \tilde{y}+\beta^{2} q^{2}=0$.

here, the solution is given by

$$
\begin{aligned}
& \tilde{y}=2 \sqrt{\frac{\beta^{2}}{3}\left(r_{h}^{4}+\frac{q^{2}}{r_{h}^{2}}\right)} \\
& \cos \left(\frac{1}{3} \cos ^{-1}\left(\frac{-3}{2 \sqrt{\frac{\beta^{2}}{3}\left(r_{h}^{4}+\frac{q^{2}}{r_{h}^{2}}\right)}\left(\frac{r_{h}^{4}}{q^{2}}+\frac{1}{r_{h}^{2}}\right)}\right)\right)
\end{aligned}
$$

One also finds the solution of (13) in [29] in which the effects of charge and finite 't Hooft coupling correction on the drag 
force are investigated. We express the drag force in terms of $T$ and $\mu$ as follows:
As one expects, the first term is the drag force at zero temperature in (20). It is clear the first correction increases the drag

$$
\begin{aligned}
& \tilde{F}=-\frac{\sqrt{\lambda}}{\pi L^{4}} v \sqrt{\frac{\beta^{2}}{3}\left(L^{2} \pi T+\sqrt{L^{4} \pi^{2} T^{2}+2 n^{2} \mu^{2}}\right)^{2}\left(n^{2} \mu^{2}+\frac{1}{4}\left(L^{2} \pi T+\sqrt{L^{4} \pi^{2} T^{2}+2 n^{2} \mu^{2}}\right)^{2}\right)} \\
& \times \cos \left(\frac{1}{3} \cos ^{-1}\left(\frac{-3 \sqrt{3} n^{2} \beta^{2} \mu^{2}\left(L^{2} \pi T+\sqrt{L^{4} \pi^{2} T^{2}+2 n^{2} \mu^{2}}\right)^{4}}{4\left(\beta^{2}\left(L^{2} \pi T+\sqrt{L^{4} \pi^{2} T^{2}+2 n^{2} \mu^{2}}\right)^{2}\left(n^{2} \mu^{2}+\frac{1}{4}\left(L^{2} \pi T+\sqrt{L^{4} \pi^{2} T^{2}+2 n^{2} \mu^{2}}\right)^{2}\right)\right)^{3 / 2}}\right)\right) .
\end{aligned}
$$

We used $r_{h}=\frac{1}{2}\left(L^{2} \pi T+\sqrt{L^{4} \pi^{2} T^{2}+2 n^{2} \mu^{2}}\right)$. Also, $\alpha^{\prime}=L^{2} / \sqrt{\lambda}$ where $\lambda$ is 't Hooft coupling. One may fix the parameters $T, L, n, \lambda$ and $\mu$ to study how the drag force on the moving particle depends on these parameters. We find that at fixed value of $\mu$, increasing $T$ leads to increasing the drag force. Also at fixed $T$, by increasing $\mu$ the drag force increases.

In the case of extremal background, one should solve the following equation:

$y^{3}-3 r_{h}^{4} \beta^{2} y+2 r_{h}^{6} \beta^{2}=0$,

one finds three real roots which can be written in the following form:

$y_{1}=2 r_{h}^{2} \beta \cos \left(\frac{\theta}{3}\right)$,

$y_{2}=2 r_{h}^{2} \beta \cos \left(\frac{2 \pi+\theta}{3}\right)$,

$y_{3}=2 r_{h}^{2} \beta \cos \left(\frac{4 \pi+\theta}{3}\right)$,

here $\theta=\cos ^{-1}\left(-\beta^{-1}\right)$. One should notice that $0<\beta^{-1}<$ 1 . By studying these roots one concludes that $y_{2}$ is not physically valid and $y_{1}$ and $y_{3}$ lead to the same results. Using $2 r_{h}^{2}=\mu^{2} n^{2}$, the drag force in the extremal background is given by

$F=-\frac{\sqrt{\lambda}}{2 \pi L^{4}} \frac{v \mu^{2} n^{2}}{\sqrt{1-v^{2}}} \cos \left(\frac{\theta}{3}\right)$.

This result can be achieved by sending $T \rightarrow 0$ in (15). By expanding (20) for small velocities, it turns out the drag force in extremal background is proportional to $v$ and $v^{3}$ for first and second order of the expansion, respectively.

It could be interesting to compare analytically the drag force at zero and finite temperature. We consider the drag force at finite temperature in (15) and expand it for small values of $T$. One finds

$\tilde{F}=F+\left(\frac{2 \sqrt{2} \pi}{3} \frac{1}{n \mu}\right) F T+\mathcal{O}\left(T^{2}\right)$ force. Thus, one finds that at zero temperature, the moving particle experiences the minimum drag force and by turning on the temperature particle feels a stronger drag force.

\section{Quasinormal modes}

Now, we consider decelerating of the probe at zero temperature and finite density. Then we should study curved open string on the late time and low velocity and consider small perturbations to the classical open string and find quasinormal modes on the string world-sheet. These modes give important information about the return to equilibrium of the trailing string after small perturbations. Therefore, one finds the friction coefficient term, $\tilde{\mu}$, in the small velocity limit. The quasinormal mode analysis in three dimensions and finite temperature has been studied in [25].

In the linear analysis, $\dot{x}$ and $x^{\prime}$ are very small and one can consider only linear terms in the equation of motion of the string. Assuming time-dependent solution as $x(t)=e^{-\tilde{\mu} t}$, the equation of motion becomes

$f(r) \partial_{r}\left(\frac{r^{4}}{L^{4}} f(r) x^{\prime}\right)=\tilde{\mu}^{2} x$.

In the case of extremal black holes, one can expand $f(r)$ close to the horizon as $f^{\prime \prime}\left(r_{h}\right)\left(r-r_{h}\right)^{2}+\mathcal{O}\left(r-r_{h}\right)^{3}$ where we can set $n^{2}$ such that $f^{\prime \prime}\left(r_{h}\right)=\mu^{-2}$ and obtain the solution

$x=c_{1} e^{\frac{\mu^{2} \tilde{\mu}}{r_{h}^{2}\left(r-r_{h}\right)}}+c_{2} e^{-\frac{\mu^{2} \tilde{\mu}}{r_{h}^{2}\left(r-r_{h}\right)}}$

which due to out-going boundary conditions we take the first part as the solution [30]. It would be interesting to compare (23) with non-extremal case in [31] as follows:

$x_{N}=c_{1}\left(r-r_{h}\right)^{\frac{\tilde{\mu}}{4 \pi T}}+c_{2}\left(r-r_{h}\right)^{-\frac{\tilde{\mu}}{4 \pi T}}$. 
As seen, two solutions are not the same. By expanding $x$ in (23), we arrive at

$x=c_{1}\left(1+\frac{\mu^{2} \tilde{\mu}}{r_{h}^{2}\left(r-r_{h}\right)}+\mathcal{O}\left(\mu^{4}\right)\right)$.

Taking derivative of $x$ yields $x^{\prime} \approx-\tilde{\mu} \mu^{2} /\left(r_{h}^{2}\left(r-r_{h}\right)^{2}\right)$ while for the non-extremal case the shape of the string is obtained as $x_{N}^{\prime} \approx-\tilde{\mu} /\left(4 \pi T\left(r-r_{h}\right)\right)$. As is clear, the near horizon behavior of trailing string in extremal black holes differs from that of the non-extremal case. In the former, it is proportional to $\left(r-r_{h}\right)^{-1}$ while in the latter it has logarithmic divergence. By studying the near horizon geometry of trailing string in the bulk, one finds that the IR divergence does not show the logarithmic behavior in the extremal case. This would be a general property of extremal backgrounds. In general, one may also consider an exponential damping solution of the following form:

$x(r, t)=\mathcal{A}(r) e^{-\tilde{\mu} t}$.

which means that $\dot{x}=-\tilde{\mu} x$. The equation of motion becomes the eigenvalue equation

$\mathcal{O} \mathcal{A}(r)=\tilde{\mu}^{2} \mathcal{A}(r), \quad \mathcal{O}=f(r) \frac{d}{d r}\left(r^{4} f(r) \frac{d}{d r}\right)$.

We have chosen $L=1$. For small $\tilde{\mu}$, we expand $x$ as a power series $x=x_{0}+\tilde{\mu}^{2} x_{1}+\cdots$. The eigenvalue equation implies that

$\mathcal{O} x=\tilde{\mu}^{2} x$.

As a result, $\mathcal{O} x_{0}=0$ and $\mathcal{O} x_{1}=x_{0}$. The general linear analysis and some universal features of studying quasinormal modes have been studied in [31]. However, for extremal black holes the near horizon behavior is not the same as the non-extremal case. For large $r_{0}$ and near $r=r_{0}$, $f(r)$ goes to 1 . Therefore, by taking $x_{0}=A$, which is a constant, and using (27) and (28) one finds for $r \sim r_{h}$

$x^{\prime} \approx \frac{-A \mu^{2} \tilde{\mu}^{2}}{r_{h}^{4}\left(r-r_{h}\right)^{2}}\left(r_{0}+\frac{\mu^{2}}{\left(r-r_{h}\right)}\right)$.

Hence we can find the friction coefficient

$\tilde{\mu}=\frac{r_{h}^{2}}{r_{0}}$,

so that, the momentum flowing down the string is

$\frac{d p}{d t}=-\tilde{\mu} p=-\frac{1}{2 \pi \alpha^{\prime}} v r_{h}^{2}$

where $m=r_{0} / 2 \pi \alpha^{\prime}$ for large $r_{0}$ limit. This is exactly the result of (12) if we expand $r_{c}$ around $r_{h}$ for small velocities.

\subsection{Stochastic behavior}

We calculate stochastic forces on the moving heavy point probe with constant velocity. One finds from holography that the stochastic forces depend on the existence of the horizon on the string world-sheet. The position of this horizon is identical with the critical point, $r_{c}$. The stochastic forces are analogous to the Brownian motion of the probe which can be found by the generalized Langevin equations. These equations involve Langevin coefficients which are proportional to the temperature of the string world-sheet. This temperature is given by the following formula [32]

$T_{w s}^{2}=\frac{1}{16 \pi^{2}}\left|\frac{1}{g_{t t} g_{r r}}\left(g_{t t} g_{x x}\right)^{\prime}\left(\frac{g_{t t}}{g_{x x}}\right)^{\prime}\right|_{r=r_{c}}$.

After replacing metric functions from (2) and considering the extremal root in (17), one finds

$$
\begin{aligned}
T_{w s}= & \frac{\sqrt{3} n \mu}{8 \pi L^{2}} \beta^{-5}\left(\sec \left(\frac{\cos ^{-1}(\theta)}{3}\right)\left(-2 \beta+\sec \left(\frac{\cos ^{-1}(\theta)}{3}\right)\right)^{2}\right. \\
& \left.\left(4 \beta^{2}+2 \beta \sec \left(\frac{\cos ^{-1}(\theta)}{3}\right)+\sec \left(\frac{\cos ^{-1}(\theta)}{3}\right)^{2}\right)\right)^{1 / 2} .
\end{aligned}
$$

For static particle when $v=0$ or $\beta=1$, one finds that $r_{c}=r_{h}$. In this case, the world-sheet temperature is exactly the Hawking temperature of the black hole. For extremal backgrounds, $T_{w s}$ vanishes when $\beta \rightarrow 1$.

The Langevin coefficients can also be found from the background metric functions as follows:

$\kappa_{T}=\left.\frac{1}{\pi \alpha^{\prime}} g_{k k}\right|_{r_{c}} T_{w s}=\frac{\sqrt{\lambda}}{\pi L^{4}} n^{2} \beta \mu^{2} \cos \left(\frac{\cos ^{-1}(\theta)}{3}\right) T_{w s}$,

here the index $k$ in $g_{k k}$ denotes a particular transverse direction of motion $x$, which is $x^{2}$ or $x^{3}$ coordinates. In the longitudinal direction it is given by

$$
\begin{aligned}
\kappa_{L}= & \left.\frac{1}{\pi \alpha^{\prime}} \frac{\left(g_{t t} g_{x x}\right)^{\prime}}{g_{x x}\left(\frac{g_{t t}}{g_{x x}}\right)^{\prime}}\right|_{r=r_{c}} T_{w s} \\
= & \frac{\sqrt{\lambda}}{3 \pi L^{4}} n^{2} \beta \mu^{2} \cos \left(\frac{\cos ^{-1}(\theta)}{3}\right)\left(1+2 \beta \cos \left(\frac{\cos ^{-1}(\theta)}{3}\right)\right. \\
& \left.+4 \beta^{2} \cos \left(\frac{\cos ^{-1}(\theta)}{3}\right)^{2}\right) T_{w s} .
\end{aligned}
$$

From the expressions (34) and (35), one derives the following ratio:

$$
\begin{aligned}
\frac{\kappa_{L}}{\kappa_{T}} & =\frac{1}{3}\left(1+\frac{4 r_{c}^{2}}{n^{4} \mu^{4}}+\frac{2 r_{c}^{2}}{n^{2} \mu^{2}}\right) \\
& =\frac{1}{3}\left(1+2 \beta \cos \left(\frac{\cos ^{-1}(\theta)}{3}\right)\right.
\end{aligned}
$$




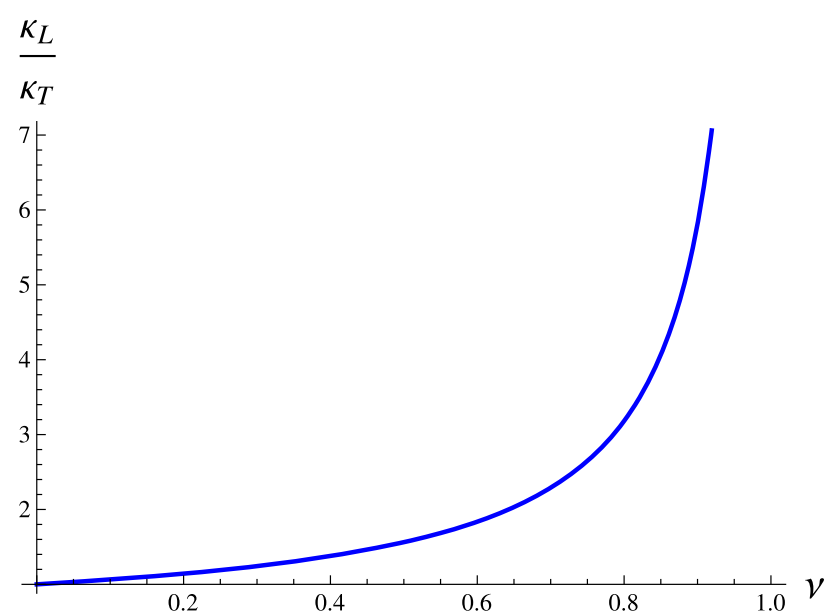

Fig. 1 The ratio $\kappa_{L} / \kappa_{T}$ in terms of the velocity of a moving particle in the extremal AdSRN background

$$
\left.+4 \beta^{2} \cos \left(\frac{\cos ^{-1}(\theta)}{3}\right)^{2}\right) .
$$

We note that the RHS of (36) is always larger than unity. For example, this can be seen by expanding the ratio at small velocity

$$
\frac{\kappa_{L}}{\kappa_{T}}=1+\frac{v}{\sqrt{3}}+\frac{17 v^{3}}{18 \sqrt{3}}+\ldots
$$

In Fig. 1, we plot $\kappa_{L} / \kappa_{T}$ quantity in terms of velocity of the heavy probe for the extremal case. It increases monotonically by increasing $v$. As one finds from (37), the ratio begins from unity. Interestingly, we find that these coefficients satisfy the inequality $\kappa_{L}>\kappa_{T}$. This universal inequality between the longitudinal and the transverse Langevin coefficients has been studied in [35] and it was discussed that the non-extremal AdSRN backgrounds satisfy this inequality. They study how $\kappa_{L} / \kappa_{T}$ changes from horizon to the boundary by considering the metric functions in (2). Here, we conclude that in the extremal AdSRN background $\kappa_{L}>\kappa_{T}$.

\section{Energy loss from 5-dimensional extremal 3-charge black holes}

The $N=4$ SYM theory is dual to type IIB supergravity on $A d S_{5} \times S^{5}$ which has a rank $3 \mathrm{SO}(6)$ R-symmetry associated to $S^{5}$ and correspond to 3 distinct chemical potentials. Indeed, this theory at nonzero temperature and chemical potential is dual to the near horizon limit of rotating D3-background consisting of a Kerr black hole with $A_{d} S_{5} \times S^{5}$ asymptotics. The theory has three different charges referred to as 3-charge black hole (3QBH). For simplicity, we can take two of them equal, i.e. $(2+1) \mathrm{QBH}$. In this case if $q_{1}=0,(2+1) \mathrm{QBH}$ is called $2 \mathrm{QBH}$.
The effective supergravity Lagrangian is given by [17]

$$
\begin{aligned}
e^{-1} \mathcal{L}= & R-\frac{1}{2}(\partial \phi)^{2}+\frac{8}{L^{2}} e^{\frac{\phi}{\sqrt{6}}}+\frac{4}{L^{2}} e^{\frac{-2 \phi}{\sqrt{6}}} \\
& -e^{\frac{-4 \phi}{\sqrt{6}}} f_{\mu \nu} f^{\mu \nu}-2 e^{\frac{2 \phi}{\sqrt{6}}} F_{\mu \nu} F^{\mu \nu} \\
& -2 \epsilon^{\mu \nu \rho \sigma \tau} f_{\mu \nu} F_{\rho \sigma} A_{\tau},
\end{aligned}
$$

where $A_{\mu}$ is the gauge field related to the two equal charges and $a_{\mu}$ is associated with the other charge. Five-dimensional black brane solution of (38) yields the metric of background

$d s^{2}=e^{2 A(r)}\left(-h(r) d t^{2}+d \vec{x}^{2}\right)+\frac{e^{2 B(r)}}{h(r)} d r^{2}$,

where

$A(r)=\log \frac{r}{L}+\frac{1}{6} \log \left(1+\frac{q_{1}^{2}}{r^{2}}\right)+\frac{1}{3} \log \left(1+\frac{q_{2}^{2}}{r^{2}}\right)$,

$B(r)=-\log \frac{r}{L}-\frac{1}{3} \log \left(1+\frac{q_{1}^{2}}{r^{2}}\right)-\frac{2}{3} \log \left(1+\frac{q_{2}^{2}}{r^{2}}\right)$,

$h(r)=1-\frac{r^{2}\left(r_{h}^{2}+q_{1}^{2}\right)\left(r_{h}^{2}+q_{2}^{2}\right)^{2}}{r_{h}^{2}\left(r^{2}+q_{1}^{2}\right)\left(r^{2}+q_{2}^{2}\right)^{2}}$.

Temperature, entropy and chemical potentials are given by the following relations:

$$
\begin{aligned}
T & =\frac{2 r_{h}^{4}+q_{1}^{2} r_{h}^{2}-q_{1}^{2} q_{2}^{2}}{2 \pi L^{2} r_{h}^{2} \sqrt{r_{h}^{2}+q_{1}^{2}}}, \\
S & =\frac{1}{4 G L^{3}} \sqrt{r_{h}^{2}+q_{1}^{2}}\left(r_{h}^{2}+q_{2}^{2}\right), \\
\mu_{1} & =\frac{q_{1}\left(r_{h}^{2}+q_{2}^{2}\right)}{L^{2} r_{h} \sqrt{r_{h}^{2}+q_{1}^{2}}}, \quad \mu_{2}=\frac{\sqrt{2} q_{2} \sqrt{r_{h}^{2}+q_{1}^{2}}}{L^{2} r_{h}} .
\end{aligned}
$$

We study the drag force on a moving particle in the background of (40). Defining $X^{\mu}=(t, r, x(r))$, since we assume the stationary motion of the heavy probe, from equation of motion we can find

$x^{\prime 2}=\frac{\left(e^{2(A+B)}\left(1-\frac{v^{2}}{h(r)}\right)\right) \Pi^{2}}{\left(h(r) e^{4 A}-\Pi^{2}\right) h(r) e^{4 A}}$,

where $\Pi$ is a constant of motion and one finds that $\Pi=$ $v \exp \left(2 A\left(r_{c}\right)\right)$. Therefore, the drag force is

$F=-\frac{1}{2 \pi \alpha^{\prime}} v e^{2 A\left(r_{c}\right)}$.

To find $r_{c}$ for non-extremal $(2+1) \mathrm{QBH}$ case, one should solve the following equation:

$$
\begin{aligned}
\left(1-v^{2}\right) & r_{h}^{2}\left(r_{c}^{2}+q_{1}^{2}\right)\left(r_{c}^{2}+q_{2}^{2}\right)^{2} \\
& -r_{c}^{2}\left(r_{h}^{2}+q_{1}^{2}\right)\left(r_{h}^{2}+q_{2}^{2}\right)^{2}=0 .
\end{aligned}
$$




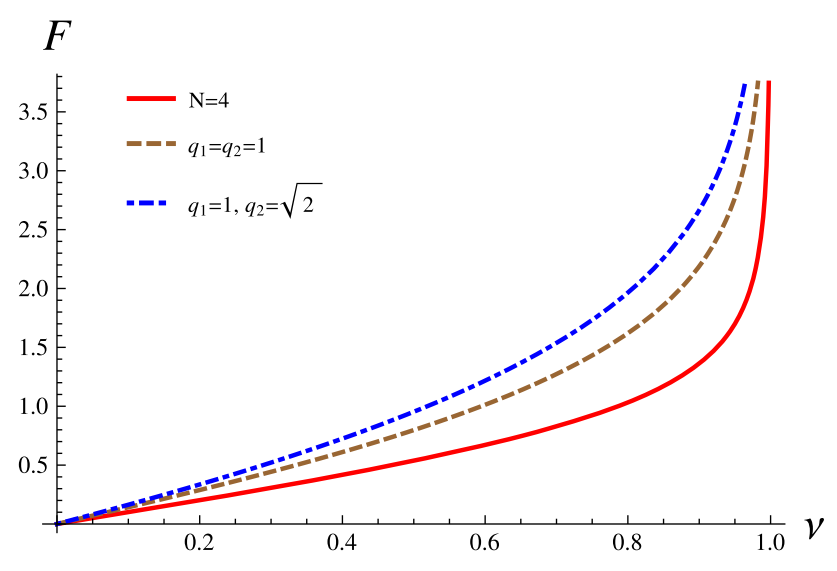

Fig. 2 We plot the drag force versus the velocity of the probe in the non-extremal $(2+1) \mathrm{QBH}$ background. We assumed $r_{h}=1.0$ and show case of $\left(q_{1}=1, q_{2}=1\right)$ with dashed line and $\left(q_{1}=1, q_{2}=\sqrt{2}\right)$ with dotdashed line

Then one can obtain the related drag force from (46). We assume $r_{h}=1$ and $L=1$. First we consider $q_{1}=q_{2}=0$, i.e. $N=4$ SYM plasma. The solution of (47) is easily obtained, i.e. $r_{c}=\beta$ and the drag force is given by

$F_{N=4}=-\frac{\pi \sqrt{\lambda}}{2} T^{2} \frac{v}{\sqrt{1-v^{2}}}$.

Next, we turn on the chemical potentials and consider two different values for $q_{1}$ and $q_{2}$. As seen from Fig. 2, we plotted the drag force as a function of velocity. From right to left, $N=$ $4,\left(q_{1}=1, q_{2}=1\right)$ and $\left(q_{1}=1, q_{2}=\sqrt{2}\right)$, respectively. One finds that considering charge density leads to increasing the force on the moving heavy particle.

\subsection{QBH extremal limits}

There are two different approaches for obtaining extremal $2 \mathrm{QBH}$. For the first approach, type I, one can set $q_{1} \rightarrow 0$ then consider $r_{h} \rightarrow 0$ to find extremal case. The second one, type II, is achieving by considering $q_{1}$ as a function of $r_{h}$ and $q_{2}$ then imposing $r_{h} \rightarrow 0$. These two limits are not exactly the same and quantities like chemical potential take different values in these approaches.

We investigate how the drag force for extremal $2 \mathrm{QBH}$ case depends on these different limits. In the type I case, after applying $q_{1} \rightarrow 0$ one finds

$$
\begin{aligned}
& A=\log \frac{r}{L}+\frac{1}{3} \log \left(1+\frac{q_{2}^{2}}{r^{2}}\right), \\
& B=-\log \frac{r}{L}-\frac{2}{3} \log \left(1+\frac{q_{2}^{2}}{r^{2}}\right), \\
& h=1-\frac{q_{2}^{4}}{\left(r^{2}+q_{2}^{2}\right)^{2}}, \quad S \rightarrow 0, \quad \mu_{1} \rightarrow 0,
\end{aligned}
$$

$\mu_{2}=\frac{\sqrt{2} q_{2}}{L^{2}}, r_{c}^{2}=q_{2}^{2}\left(-1+\frac{1}{\sqrt{1-v^{2}}}\right)$.

where $r_{c}$ is found from $h\left(r_{c}\right)-v^{2}=0$ equation. Therefore, one finds the drag force as

$$
\begin{aligned}
F & =-\frac{v}{2 \pi \alpha^{\prime} L^{2}} \frac{q_{2}^{2}\left(1-\sqrt{1-v^{2}}\right)^{1 / 3}}{\sqrt{1-v^{2}}} \\
& =-\frac{v \mu_{2}^{2} \sqrt{\lambda}}{4 \pi} \frac{\left(1-\sqrt{1-v^{2}}\right)^{1 / 3}}{\sqrt{1-v^{2}}} .
\end{aligned}
$$

For type II extremal 2QBH, using $T=0$, we obtain $q_{1}^{2}=$ $2 r_{h}^{4} /\left(q_{2}^{2}-r_{h}^{2}\right)$ which is extremal $(2+1) \mathrm{QBH}$ case. By plugging $q_{1}^{2}$ into the relevant equations we find

$$
\begin{aligned}
A & =\log \frac{r}{L}+\frac{1}{6} \log \left(1+\frac{2 r_{h}^{4}}{q_{2}^{2}-r^{2}}\right)+\frac{1}{3} \log \left(1+\frac{q_{2}^{2}}{r^{2}}\right), \\
B & =-\log \frac{r}{L}-\frac{1}{3} \log \left(1+\frac{2 r_{h}^{4}}{q_{2}^{2}-r^{2}}\right)-\frac{2}{3} \log \left(1+\frac{q_{2}^{2}}{r^{2}}\right), \\
h & =1-\frac{r^{2}\left(1+\frac{2 r_{h}^{2}}{q_{2}^{2}-r_{h}^{2}}\right)\left(r_{h}^{2}+q_{2}^{2}\right)^{2}}{\left(r^{2}+\frac{2 r_{h}^{4}}{q_{2}^{2}-r_{h}^{2}}\right)\left(r^{2}+q_{2}^{2}\right)^{2}}, \\
S & =\frac{1}{4 G L^{3}} \frac{r_{h}^{2}\left(q_{2}^{2}+r_{h}^{2}\right)^{3 / 2}}{\left(q_{2}^{2}-r_{h}^{2}\right)^{1 / 2}}, \\
\mu_{1} & =\frac{\sqrt{2}\left(r_{h}^{2}+q_{2}^{2}\right)}{\sqrt{\left(q_{2}^{2}-r_{h}^{2}\right)\left(1+\frac{2 r_{h}^{2}}{q_{2}^{2}-r_{h}^{2}}\right)}}, \\
\mu_{2}= & \frac{\sqrt{2} q_{2} \sqrt{1+\frac{2 r_{h}^{2}}{q_{2}^{2}-r_{h}^{2}}}}{L^{2}}
\end{aligned}
$$

Now, one should impose $r_{h} \rightarrow 0$ to find metric functions and $r_{c}$. One finds that in type II and type I the metric functions $A, B$, and $r_{c}$ does not change and $\mu_{1}=\mu_{2}=\sqrt{2} q_{2} / L^{2}$. Also, entropy in both limits vanishes. As a quick result, according to (45), the shape of string in the bulk for these two limits would be the same since $A$ and $B$ as well as $r_{c}$ does not change in these limits. Finally, the drag force for type II extremal $2 \mathrm{QBH}$ would be

$$
F=-\frac{v \mu_{1}^{2} \sqrt{\lambda}}{4 \pi} \frac{\left(1-\sqrt{1-v^{2}}\right)^{1 / 3}}{\sqrt{1-v^{2}}}
$$

The drag force in non-extremal five dimensional backgrounds with three different charges has been studied in [36].

Comparing the drag force in two extremal limits, one finds that they are the same, i.e. the drag force for extremal $2 \mathrm{QBH}$ cannot be affected from these two limits.

One may also compare the drag force for extremal $2 \mathrm{QBH}$ in (55) with extremal AdSRN result given in (20). Notice in 
extremal 2QBH background entropy vanishes while in the extremal AdSRN background it is finite. Then two mediums are at zero temperature and finite density while mediums do not have the same entropy. Considering a moving particle in two mediums, one finds different equations for the drag force. As an important observation, one finds that the drag force in both extremal backgrounds depends on the squared of the chemical potential. However, the dependency of the equations to the velocity of the particle is not the same as each other

\subsection{Stochastic behavior}

In this section, we study the Brownian motion of a moving heavy particle at zero temperature and finite density with zero entropy. Comparing with extremal AdSRN background, the motivation is to find how $T_{w s}, \kappa_{L}$ and $\kappa_{T}$ change in extremal $2 \mathrm{QBH}$ geometry. We investigate the dependence of these quantities on the type of the background.

From (39), we obtain the temperature of the word-sheet trailing string in this background

$$
\begin{aligned}
T_{w s}^{2} & =\left.\frac{1}{16 \pi^{2}}\left(e^{2(A(r)-B(r))} h^{\prime}(r)\left(4 A^{\prime}(r) h(r)+h^{\prime}(r)\right)\right)\right|_{r=r_{c}} \\
& =\frac{q_{2}^{2}(-1+\beta)\left(1+\beta+3 \beta^{2}\right)}{3 L^{4} \pi^{2} \beta^{4}} .
\end{aligned}
$$

Here, we used $r_{c}^{2}=q_{2}^{2}(\beta-1)$. Notice $r_{c}$ does not change in type I and II limits.

The Langevin coefficients can be calculated analytically. For the transverse direction, the coefficient is

$$
\begin{aligned}
\kappa_{T} & =\frac{1}{\pi \alpha^{\prime}} e^{2 A\left(r_{c}\right)} T_{w s} \\
& =\frac{\sqrt{\lambda}}{L^{6} \sqrt{3} \pi^{2}} \frac{q_{2}^{3} \sqrt{-1-2 \beta^{2}+3 \beta^{3}}}{\beta\left(\frac{\beta}{\beta-1}\right)^{1 / 3}} .
\end{aligned}
$$

The longitudinal coefficient is given by

$$
\begin{aligned}
\kappa_{L} & =\frac{1}{\pi \alpha^{\prime}} \frac{e^{2 A\left(r_{c}\right)}\left(4 A^{\prime}\left(r_{c}\right) h\left(r_{c}\right)+h^{\prime}\left(r_{c}\right)\right)}{h^{\prime}\left(r_{c}\right)} T_{w s} \\
& =\frac{\sqrt{\lambda}}{L^{4} 3 \sqrt{3} \pi^{2}} \frac{q_{2}^{3}\left(1+\beta+3 \beta^{2}\right) \sqrt{-1-2 \beta^{2}+3 \beta^{3}}}{\beta\left(\frac{\beta}{\beta-1}\right)^{1 / 3}} .
\end{aligned}
$$

We analyze the universal inequality $\kappa_{L}>\kappa_{T}$ in the extremal 2QBH background. One finds

$$
\frac{\kappa_{L}}{\kappa_{T}}=\frac{1}{3}\left(1+\beta+3 \beta^{2}\right) \text {. }
$$

In Fig. 3, we show the ratio of longitudinal to transverse coefficient $\frac{\kappa_{L}}{\kappa_{T}}$ versus the velocity for two different extremal backgrounds, i.e. extremal 2QBH and extremal AdSRN,

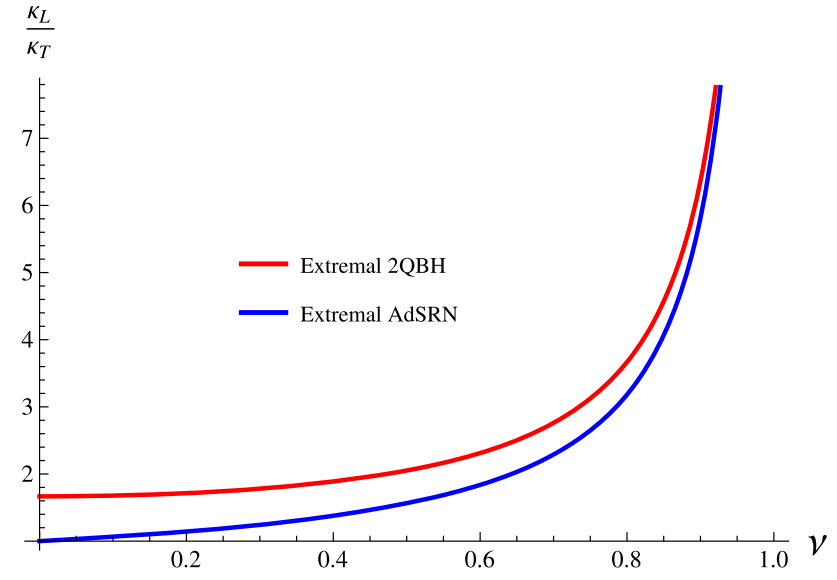

Fig. 3 The ratio $\frac{\kappa_{L}}{\kappa_{T}}$ as a function of the velocity for different extremal backgrounds. From top to down, extremal 2QBH and extremal AdSRN backgrounds

respectively. As we explained extremal 2QBH corresponds to a medium at finite density with zero temperature and zero entropy. While extremal AdSRN correspond to a medium at finite density with zero temperature and finite entropy. We observe that the transverse and longitudinal coefficients depend on the properties of the medium. One may study behavior of $\kappa_{L}$ and $\kappa_{T}$ in two mediums. As a general result, we find that in both cases $\kappa_{L}>\kappa_{T}$.

\section{10D case}

In this section, we consider ten-dimensional lift of 3-charge black holes as solutions of type IIB supergravity and study different extremal cases in this background. As [25,37], the metric is given by

$$
\begin{aligned}
d s^{2}= & \sqrt{\Delta}\left(-\left(H_{1} H_{2} H_{3}\right)^{-2 / 3} h d t^{2}+\left(H_{1} H_{2} H_{3}\right)^{1 / 3}\left[h^{-1} d r^{2}\right.\right. \\
& \left.\left.+r^{2}\left(d \chi_{1}^{2}+\sin ^{2} \chi_{1}\left(d \chi_{2}^{2}+\sin ^{2} \chi_{2} d \chi_{3}^{2}\right)\right)\right]\right) \\
& +\frac{L^{2}}{\sqrt{\Delta}} \sum_{i=1}^{3} H_{i}\left(H_{1} H_{2} H_{3}\right)^{-1 / 3}\left(d \varphi_{i}^{2}\right. \\
& \left.+\varphi_{i}^{2}\left(d \psi_{i}+j^{i} d t\right)^{2}\right),
\end{aligned}
$$

here the metric functions are

$$
\begin{aligned}
\Delta & =\sum_{i=1}^{3} H_{i}\left(H_{1} H_{2} H_{3}\right)^{1 / 3} \varphi_{i}^{2}, \quad h=1-\frac{m}{r^{2}}+\frac{r^{2}}{L^{2}} H_{1} H_{2} H_{3}, \\
H_{i} & =1+\frac{q_{i}}{r^{2}}, \quad j^{i}=\frac{\left(1-H_{i}^{-1}\right) \sqrt{q_{i}\left(m+q_{i}\right)}}{L q_{i}} \\
\varphi_{1} & =\sin \theta_{1}, \quad \varphi_{2}=\cos \theta_{1} \sin \theta_{2}, \quad \varphi_{3}=\cos \theta_{1} \cos \theta_{2} .
\end{aligned}
$$


$\chi_{i}$ are coordinates on 3 -sphere and $S^{5}$ is parameterized by $\left(\theta_{1}, \theta_{2}, \psi_{1}, \psi_{2}, \psi_{3}\right)$. The Hawking temperature and entropy of the black hole are

$$
\begin{aligned}
& T=\frac{1}{2 \pi r_{h}^{2} L^{2}} \frac{2 r_{h}^{6}+r_{h}^{4}\left(q_{1}+q_{2}+q_{3}+L^{2}\right)-q_{1} q_{2} q_{3}}{\prod_{i=1}^{3} \sqrt{r_{h}^{2}+q_{i}}}, \\
& S=\frac{\pi^{2}}{2 G} \prod_{i=1}^{3} \sqrt{r_{h}^{2}+q_{i}} .
\end{aligned}
$$

here $G$ is the five-dimensional Newton's constant and horizon radius, $r_{h}$, is obtained from $h\left(r_{h}\right)=0$. For simplicity we ignore $\theta_{i}$ directions and rewrite the metric as

$$
\begin{aligned}
d s^{2}= & f\left[-\alpha d t^{2}+\beta d r^{2}+\gamma\left(d \chi_{1}^{2}+\sin ^{2} \chi_{1}\left(d \chi_{2}^{2}\right.\right.\right. \\
& \left.\left.\left.+\sin ^{2} \chi_{2} d \chi_{3}^{2}\right)\right)\right]+\frac{1}{f} \sum_{i=1}^{3} \kappa_{i}\left(d \psi_{i}+j^{i} d t\right)^{2}
\end{aligned}
$$

here

$$
\begin{aligned}
& \sqrt{\Delta} \equiv f, \quad h\left(H_{1} H_{2} H_{3}\right)^{-2 / 3} \equiv \alpha, \\
& \frac{\left(H_{1} H_{2} H_{3}\right)^{1 / 3}}{h} \equiv \beta, \\
& r^{2}\left(H_{1} H_{2} H_{3}\right)^{1 / 3} \equiv \gamma, \quad H_{i}\left(H_{1} H_{2} H_{3}\right)^{-1 / 3} L^{2} \equiv \kappa_{i} .
\end{aligned}
$$

Now, we investigate stationary solutions in which $\vartheta_{i}=\dot{\chi}_{i}$ and $\omega_{i}=\dot{\psi}_{i}$ are constant by taking static gauge $(\tau=$ $t, \sigma=r)$. Defining $X^{\mu}=\left(t, r, \chi_{1}, \chi_{2}, \chi_{3}, \psi_{1}, \psi_{2}, \psi_{3}\right)$, Lagrangian can be obtained from the string action in (7) as

$$
\begin{aligned}
X^{\prime} \dot{X}= & f \gamma\left[\vartheta_{1} \chi_{1}^{\prime}+\sin ^{2} \chi_{1}\left(\vartheta_{2} \chi_{2}^{\prime}+\sin ^{2} \chi_{2} \vartheta_{3} \chi_{3}^{\prime}\right)\right] \\
& +\frac{1}{f} \sum_{i=1}^{3} \kappa_{i}\left(\omega_{i}+j^{i}\right) \psi_{i}^{\prime} \\
X^{\prime 2}= & f \beta+f \gamma\left[\left(\chi_{1}^{\prime}\right)^{2}+\sin ^{2} \chi_{1}\left(\left(\chi_{2}^{\prime}\right)^{2}+\sin ^{2} \chi_{2}\left(\chi_{3}^{\prime}\right)^{2}\right)\right] \\
& +\frac{1}{f} \sum_{i=1}^{3} \kappa_{i} \psi_{i}^{\prime} \\
\dot{X}^{2}= & -f \alpha+f \gamma\left[\vartheta_{1}^{2}+\sin ^{2} \chi_{1}\left(\vartheta_{2}^{2}+\sin ^{2} \chi_{2} \vartheta_{3}^{2}\right)\right] \\
& +\frac{1}{f} \sum_{i=1}^{3} \kappa_{i}\left(\omega_{i}+j^{i}\right)^{2} .
\end{aligned}
$$

Relevant momenta for the string spining in $\psi_{1}=\psi$ and $\chi_{i}$ directions corresponded to associated drag forces are gained as

$$
\begin{aligned}
\Pi_{\chi_{1}}= & \frac{-1}{2 \pi \alpha^{\prime}} \frac{\partial \mathcal{L}}{\partial \chi_{1}^{\prime}}=\frac{-1}{2 \pi \alpha^{\prime} \mathcal{L}}\left[f ^ { 2 } \gamma ^ { 2 } \operatorname { s i n } ^ { 2 } \chi _ { 1 } \left(\vartheta_{1} \vartheta_{2} \chi_{2}^{\prime}\right.\right. \\
& \left.+\vartheta_{1} \vartheta_{3} \chi_{3}^{\prime} \sin ^{2} \chi_{2}\right)+\gamma \kappa_{1}\left(\omega_{1}+j^{1}\right) \vartheta_{1} \psi^{\prime}+f^{2} \alpha \gamma \chi_{1}^{\prime} \\
& -f^{2} \gamma^{2} \chi_{1}^{\prime} \sin ^{2} \chi_{1}\left(\vartheta_{2}^{2}+\sin ^{2} \chi_{2} \vartheta_{3}^{2}\right) \\
& \left.-\sum_{i=1}^{3} \gamma \kappa_{i}\left(\omega_{i}+j^{i}\right)^{2} \chi_{1}^{\prime}\right],
\end{aligned}
$$

$$
\begin{aligned}
\Pi_{\chi_{2}}= & \frac{-1}{2 \pi \alpha^{\prime}} \frac{\partial \mathcal{L}}{\partial \chi_{2}^{\prime}}=\frac{-1}{2 \pi \alpha^{\prime} \mathcal{L}}\left[\operatorname { s i n } ^ { 2 } \chi _ { 1 } \left(f ^ { 2 } \gamma ^ { 2 } \left(\vartheta_{1} \vartheta_{2} \chi_{1}^{\prime}\right.\right.\right. \\
& \left.+\vartheta_{2} \vartheta_{3} \chi_{3}^{\prime} \sin ^{2} \chi_{1} \sin ^{2} \chi_{2}\right)+\gamma \kappa_{1}\left(\omega_{1}+j^{1}\right) \vartheta_{2} \psi^{\prime} \\
& +f^{2} \alpha \gamma \chi_{2}^{\prime}-f^{2} \gamma^{2} \chi_{2}^{\prime}\left(\vartheta_{1}^{2}+\sin ^{2} \chi_{1} \sin ^{2} \chi_{2} \vartheta_{3}^{2}\right) \\
& \left.\left.-\sum_{i=1}^{3} \gamma \kappa_{i}\left(\omega_{i}+j^{i}\right)^{2} \chi_{2}^{\prime}\right)\right], \\
\Pi_{\chi_{3}}= & \frac{-1}{2 \pi \alpha^{\prime}} \frac{\partial \mathcal{L}}{\partial \chi_{3}^{\prime}}=\frac{-1}{2 \pi \alpha^{\prime} \mathcal{L}}\left[\operatorname { s i n } ^ { 2 } \chi _ { 1 } \operatorname { s i n } ^ { 2 } \chi _ { 2 } \left(f ^ { 2 } \gamma ^ { 2 } \left(\vartheta_{1} \vartheta_{3} \chi_{1}^{\prime}\right.\right.\right. \\
& \left.+\vartheta_{2} \vartheta_{3} \chi_{2}^{\prime} \sin ^{2} \chi_{1}\right)+\gamma \kappa_{1}\left(\omega_{1}+j^{1}\right) \vartheta_{3} \psi^{\prime} \\
& +f^{2} \alpha \gamma \chi_{3}^{\prime}-f^{2} \gamma^{2} \chi_{3}^{\prime}\left(\vartheta_{1}^{2}+\sin ^{2} \chi_{1} \vartheta_{2}^{2}\right) \\
& \left.\left.-\sum_{i=1}^{3} \gamma \kappa_{i}\left(\omega_{i}+j^{i}\right)^{2} \chi_{3}^{\prime}\right)\right], \\
\Pi_{\psi_{i}}= & \frac{-1}{2 \pi \alpha^{\prime}} \frac{\partial \mathcal{L}}{\partial \psi^{\prime}}=\frac{-1}{2 \pi \alpha^{\prime} \mathcal{L}}\left[\sum _ { i = 1 } ^ { 3 } \gamma \kappa _ { i } ( \omega _ { i } + j ^ { i } ) \left(\vartheta_{1} \chi_{1}^{\prime}\right.\right. \\
& \left.+\sin ^{2} \chi_{1}\left(\vartheta_{2} \chi_{2}^{\prime}+\sin ^{2} \chi_{2} \vartheta_{3} \chi_{3}^{\prime}\right)\right)+\alpha \kappa_{1} \psi^{\prime} \\
& -\gamma \kappa_{1} \psi^{\prime}\left(\vartheta_{1}^{2}+\sin ^{2} \chi_{1}\left(\vartheta_{2}^{2}+\sin ^{2} \chi_{2} \vartheta_{3}^{2}\right)\right) \\
& -\frac{1}{f^{2}} \kappa_{2} \kappa_{1}\left(\omega_{2}+j^{2}\right)^{2} \psi^{\prime} \\
& \left.-\frac{1}{f^{2}} \kappa_{3} \kappa_{1}\left(\omega_{3}+j^{3}\right)^{2} \psi^{\prime}\right] .
\end{aligned}
$$

We find from the above equations that these quantities are no longer constant and satisfy their following equation of motion

$\partial_{r}\left(\frac{\partial \mathcal{L}}{\partial X^{\prime}}\right)+\partial_{t}\left(\frac{\partial \mathcal{L}}{\partial \dot{X}}\right)-\frac{\partial \mathcal{L}}{\partial X}=0$.

To avoid this complexity, we focus on the case where $\chi_{i}=$ const. As a result, the drag force would be

$F_{\psi}=-\frac{1}{2 \pi \alpha^{\prime}} \frac{\partial \mathcal{L}}{\partial \psi^{\prime}}=-\frac{1}{2 \pi \alpha^{\prime} \mathcal{L}}\left(\frac{\kappa_{1} \psi^{\prime}}{f^{2}} I_{1}\right)$.

where $I_{1}=\alpha f^{2}-\kappa_{2}\left(j^{2}\right)^{2}-\kappa_{3}\left(j^{3}\right)^{2}$. Plugging $\mathcal{L}$ into the formula, we have $\left(\psi^{\prime}\right)^{2}=\Pi^{2} I_{2} /\left(\frac{\kappa_{1}^{2}}{f^{4}} I_{1}^{2}-\frac{\kappa_{1}(\Pi)^{2}}{f^{2}} I_{1}\right)$ where $I_{2}=\alpha \beta f^{2}-\beta\left[\kappa_{1}\left(\omega+j^{1}\right)^{2}+\kappa_{2}\left(j^{2}\right)^{2}+\kappa_{3}\left(j^{3}\right)^{2}\right]$ and $\Pi$ is a constant of motion. One should calculate drag force at the critical point, which this leads to the following relations

$$
\begin{gathered}
\alpha-\frac{\sum_{i=1}^{3} \kappa_{i}\left(\omega+j^{i}\right)^{2}}{f^{2}}=0, \\
\Pi^{2}=\frac{\kappa_{1}}{f^{2}} I_{1}=\frac{\kappa_{1}^{2}\left(\omega+j^{1}\right)^{2}}{f^{2}} .
\end{gathered}
$$

Finally, one can find the drag force as

$$
F_{\psi}=-\frac{1}{2 \pi \alpha^{\prime}} \Pi^{\prime}=-\frac{1}{2 \pi \alpha^{\prime}} \frac{\kappa_{1}\left(\omega+j^{1}\right)}{f} .
$$

Now we study the near horizon behavior of the trailing string in both non-extremal and extremal cases. In nonextremal cases, $\alpha$ and $\beta$ can be expanded as 


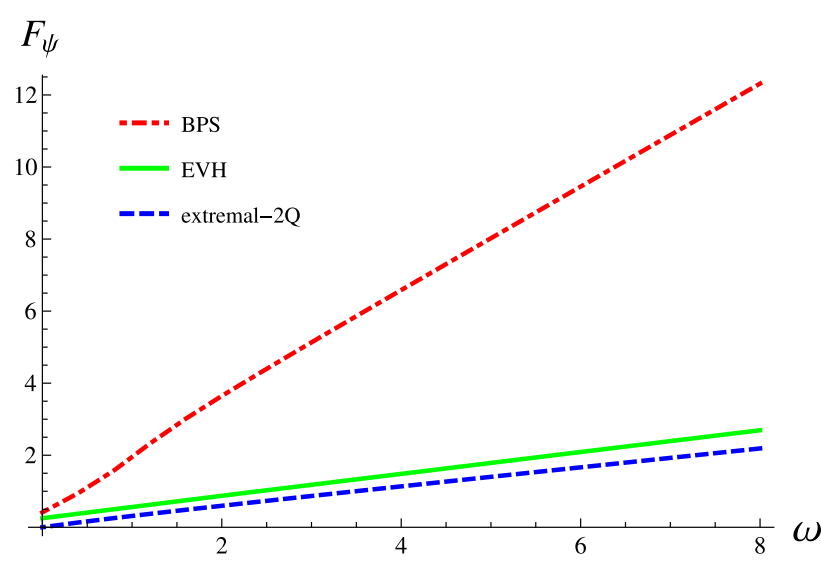

Fig. 4 The drag force for different extremal black holes with different R-charges; BPS case with $q_{1}=1, q_{2}=q_{3}=0$ and $r_{h}=0 \Rightarrow m=0$ (dotdashed), EVH case with $q_{2}=q_{3}=1$ and $r_{h}=0.5 \Rightarrow q_{1}=0.16$ and $m=3.27$ (solid line), and extremal 2-charge case with $q_{1}=$ $0, q_{2}=q_{3}=1$ and $r_{h}=0 \Rightarrow m=m_{c}=1$ (dashed). We assume $\lambda=100$

$\alpha(r)=\alpha^{\prime}\left(r_{h}\right)\left(r-r_{h}\right)+\mathcal{O}\left(r-r_{h}\right)^{2}$,

$\beta(r)=\frac{\beta_{-1}}{\left(r-r_{h}\right)}+\mathcal{O}(1)$.

By studying $\psi^{\prime 2}$, for single charge case the near horizon behavior of $\psi^{\prime}$ is proportional to $\left(r-r_{h}\right)^{-1}$ so that $\psi$ would be logarithmically divergent, whereas for 3-charge case one finds that $\psi^{\prime} \propto\left(r-r_{h}\right)^{-1 / 2}$ and there is no divergency from $\psi$. For extremal case, the expansion of $\alpha$ and $\beta$ are as follows

$\alpha(r)=\alpha^{\prime \prime}\left(r_{h}\right)\left(r-r_{h}\right)^{2}+\mathcal{O}\left(r-r_{h}\right)^{3}$,

$\beta(r)=\frac{\beta_{-2}}{\left(r-r_{h}\right)^{2}}+\mathcal{O}\left(r-r_{h}\right)^{-1}$.

In this case for single charge, $\psi^{\prime}$ in the near horizon would be proportional to $\left(r-r_{h}\right)^{-2}$. This behavior for 3-charge case would be $\psi^{\prime} \propto\left(r-r_{h}\right)^{-1}$ and it has logarithmic divergence.

As a result, one finds that in both cases of extremal and non-extremal the near horizon behavior depends on the charges of the background.

\subsection{EVH limit}

We are interested in calculating the drag force (78) for different extremal black holes with different R-charges (see Fig. 4 ). We take into account black holes with the limit of Extremal Vanishing Horizon (EVH) [38]. To find the limit of EVH for a black hole, the temperature and the entropy or related area of horizon should be set to zero while their ratio is kept finite. By vanishing the temperature, one finds

$$
\begin{aligned}
q_{1} & =\frac{r_{h}^{4} L^{2}+2 r_{h}^{6}+r_{h}^{4}\left(q_{2}+q_{3}\right)}{m_{c} L^{2}-r_{h}^{4}}=r_{h}^{4} C, \\
C & =\frac{L^{2}+2 r_{h}^{2}+\left(q_{2}+q_{3}\right)}{m_{c} L^{2}-r_{h}^{4}},
\end{aligned}
$$

where $m_{c}=q_{2} q_{3} / L^{2}$. From $h\left(r_{h}\right)=0$, we have

$m-m_{c}=2 r_{h}^{2} m_{c} C+\frac{r_{h}^{4}}{L^{2}}\left[\left(q_{2}+q_{3}\right) C-1\right]$.

We can also obtain extremal 2-charge case in two different approaches. In the first approach, one should set $q_{1}$ and $r_{h}$ to zero, taking $q_{2}=q_{3}$. For the second approach, one can consider $q_{1}$ as a function of $r_{h}$ and $q_{2}$ as the relation obtained in (81). By tending $r_{h}$ to zero, we arrive at the case. By putting these quantities into (78), one finds that these two approaches would arrive at the same result for the drag force equation.

Another case we would like to study is BPS single charge. This one is an extremal black hole gained by $q_{2}=q_{3}=0$ which leads to $m=0$. The drag force is computed for each of the extremal cases we discussed as a function of $\omega$ in Fig. 4. As it is clear from this figure, increasing $\omega$ leads to increasing the drag force.

\section{Conclusion}

In this paper, we studied the drag force on a moving heavy point-like probe in a strongly-coupled medium at zero temperature and finite density. First, we considered the AdSRN black brane background and calculated the drag force, analytically. One important observation is that unlike [27], we do not find any critical value for the velocity of the probe from the drag force relation. Also using the holographic method, we investigated the near horizon behavior of the trailing string in the bulk of the extremal AdSRN background. Interestingly, in contrast with the non-extremal case, there is no logarithmic divergency. We also studied the stochastic behavior of the probe in this medium. We computed the temperature of the world-sheet for the extremal case. The Langevin coefficients were computed and it is found that they satisfy the universal predicted bound, i.e. $\kappa_{L} / \kappa_{T}>1$.

Next, we generalized these calculations to 5-dimensional 3QBH geometry, considering vanishing entropy limit. There are two different approaches to get extremal 2QBH geometries. It is shown that the drag force formula in these approaches does not change. We also computed the Langevin coefficients in the extremal case and showed that the ratio $\kappa_{L} / \kappa_{T}$ obeys the universal bound.

It is worthwhile to mention that in extremal $2 \mathrm{QBH}$ background entropy vanishes while in the extremal AdSRN background it is finite. Then two corresponding mediums are not the same. The drag force on a moving particle in two mediums have different equations. As an important observation, one finds that the drag force in both extremal backgrounds depends on the squared of the chemical potential. However, the dependency of the equations to the velocity of the particle is not the same as each other. We observe also that the transverse and longitudinal coefficients differ in two medi- 
ums. One may study how $\kappa_{L}$ and $\kappa_{T}$ change in two mediums. As a general result, we find that in both cases $\kappa_{L}>\kappa_{T}$.

Finally, we investigated the drag force in 10-dimensional $3 \mathrm{QBH}$ and considered the motion of string in $S^{5}$ part directions. We studied the near horizon behavior of the trailing string in non-extremal and extremal cases and discussed how the logarithmic divergency depends on the charges of the background. The drag force has been calculated numerically for EVH, extremal 2-charge and BPS single charge cases. It was shown that by increasing the angular velocity the drag force also increases.

Acknowledgements The authors would like to acknowledge the scientific atmosphere of school of physics of IPM, Tehran, during the course of this project.

Open Access This article is distributed under the terms of the Creative Commons Attribution 4.0 International License (http://creativecomm ons.org/licenses/by/4.0/), which permits unrestricted use, distribution, and reproduction in any medium, provided you give appropriate credit to the original author(s) and the source, provide a link to the Creative Commons license, and indicate if changes were made. Funded by SCOAP ${ }^{3}$.

\section{References}

1. J.M. Maldacena, The large $\mathrm{N}$ limit of superconformal field theories and supergravity. Int. J. Theor. Phys. 38, 1113 (1999). arXiv:hep-th/9711200

2. J.M. Maldacena, Adv. Theor. Math. Phys. 2, 231 (1998)

3. S.S. Gubser, I.R. Klebanov, A.M. Polyakov, Gauge theory correlators from noncritical string theory. Phys. Lett. B 428, 105 (1998). arXiv:hep-th/9802109

4. E. Witten, Anti-de Sitter space and holography. Adv. Theor. Math. Phys. 2, 253 (1998). arXiv:hep-th/9802150

5. J.M. Bardeen, G.T. Horowitz, The extreme Kerr throat geometry: a vacuum analog of $\operatorname{AdS}(2)$ x S**2. Phys. Rev. D 60, 104030 (1999). arXiv:hep-th/9905099

6. A. Strominger, C. Vafa, Microscopic origin of the BekensteinHawking entropy. Phys. Lett. B 379, 99 (1996). hep-th/9601029

7. T. Faulkner, H. Liu, J. McGreevy, D. Vegh, Emergent quantum criticality, Fermi surfaces, and AdS(2). Phys. Rev. D 83, 125002 (2011). arXiv:0907.2694 [hep-th]

8. M. Edalati, J.I. Jottar, R.G. Leigh, Transport coefficients at zero temperature from extremal black holes. JHEP 1001, 018 (2010). arXiv:0910.0645 [hep-th]

9. S.K. Chakrabarti, S. Jain, S. Mukherji, Viscosity to entropy ratio at extremality. JHEP 1001, 068 (2010). arXiv:0910.5132 [hep-th]

10. R.G. Cai, Y. Liu, Y.W. Sun, Transport coefficients from extremal Gauss-Bonnet black holes. JHEP 1004, 090 (2010). arXiv:0910.4705 [hep-th]

11. M.F. Paulos, Transport coefficients, membrane couplings and universality at extremality. JHEP 1002, 067 (2010). arXiv:0910.4602 [hep-th]

12. S. Sadeghian, M.M. Sheikh-Jabbari, M.H. Vahidinia, H. Yavartanoo, Near horizon structure of extremal vanishing horizon black holes. Nucl. Phys. B 900, 222 (2015). arXiv:1504.03607 [hep-th]

13. M.M. Sheikh-Jabbari, H. Yavartanoo, EVH black holes, AdS3 throats and EVH/CFT proposal. JHEP 1110, 013 (2011). arXiv:1107.5705 [hep-th]
14. M. Johnstone, M .M. Sheikh-Jabbari, J. Simon, H. Yavartanoo, Extremal black holes and the first law of thermodynamics. Phys. Rev. D 88(10), 101503 (2013). arXiv:1305.3157 [hep-th]

15. K. Hajian, A. Seraj, M.M. Sheikh-Jabbari, NHEG mechanics: laws of near horizon extremal geometry (Thermo)dynamics. JHEP 1403, 014 (2014). arXiv:1310.3727 [hep-th]

16. A. Seraj, M .M. Sheikh-Jabbari, Near horizon extremal geometry perturbations: dynamical field perturbations vs. parametric variations. JHEP 1410, 111 (2014). arXiv:1407.1992 [hep-th]

17. O. DeWolfe, S.S. Gubser, C. Rosen, Fermionic response in a zero entropy state of $\mathcal{N}=4$ super-Yang-Mills. Phys. Rev. D 91(4), 046011 (2015). arXiv: 1312.7347 [hep-th]

18. O. DeWolfe, O. Henriksson, C. Rosen, Fermi surface behavior in the ABJM M2-brane theory. Phys. Rev. D 91(12), 126017 (2015). arXiv: 1410.6986 [hep-th]

19. C. Cosnier-Horeau, S.S. Gubser, Holographic Fermi surfaces at finite temperature in top-down constructions. Phys. Rev. D 91(6), 066002 (2015). arXiv:1411.5384 [hep-th]

20. H.K. Kunduri, J. Lucietti, H.S. Reall, Near-horizon symmetries of extremal black holes. Class. Quantum Gravity 24, 4169 (2007). arXiv:0705.4214 [hep-th]

21. S.A. Hartnoll, J. Polchinski, E. Silverstein, D. Tong, Towards strange metallic holography. JHEP 1004, 120 (2010). arXiv:0912.1061 [hep-th]

22. T. Matsui, H. Satz, J/ $\psi$ suppression by Quark-Gluon plasma formation. Phys. Lett. B 178, 416 (1986)

23. J. Casalderrey-Solana, H. Liu, D. Mateos, K. Rajagopal, U. A. Wiedemann, Gauge/string duality, hot QCD and heavy ion collisions, arXiv:1101.0618 [hep-th]

24. O. DeWolfe, S.S. Gubser, C. Rosen, D. Teaney, Heavy ions and string theory. Prog. Part. Nucl. Phys. 75, 86 (2014). arXiv:1304.7794 [hep-th]

25. C.P. Herzog, A. Karch, P. Kovtun, C. Kozcaz, L.G. Yaffe, Energy loss of a heavy quark moving through $\mathrm{N}=4$ supersymmetric YangMills plasma. JHEP 0607, 013 (2006). arXiv:hep-th/0605158

26. S.S. Gubser, Drag force in AdS/CFT. Phys. Rev. D 74, 126005 (2006). arXiv:hep-th/0605182

27. S.S. Gubser, A. Yarom, Pointlike probes of superstring-theoretic superfluids. JHEP 1003, 041 (2010). arXiv:0908.1392 [hep-th]

28. J.F. Fuini III, A. Karch, Energy loss calculations of moving defects for general holographic metrics. Phys. Rev. D 85, 066006 (2012). arXiv:1112.2747 [hep-th]

29. K.B. Fadafan, Charge effect and finite 't Hooft coupling correction on drag force and jet quenching parameter. Eur. Phys. J. C 68, 505 (2010). arXiv:0809.1336 [hep-th]

30. D.T. Son, A.O. Starinets, Minkowski space correlators in AdS / CFT correspondence: recipe and applications. JHEP 0209, 042 (2002). arXiv:hep-th/0205051

31. C.P. Herzog, Energy loss of heavy quarks from asymptotically AdS geometries. JHEP 0609, 032 (2006). arXiv:hep-th/0605191

32. D. Giataganas, H. Soltanpanahi, Heavy quark diffusion in strongly coupled anisotropic plasmas. JHEP 1406, 047 (2014). arXiv:1312.7474 [hep-th]

33. R. Rapp, H. van Hees, Heavy Quarks in the Quark-Gluon Plasma, R. C. Hwa, X.-N. Wang (Ed.) Quark Gluon Plasma 4, World Scientific, 111 (2010). arXiv:0903.1096 [hep-ph]

34. S. Nakamura, H. Ooguri, Out of equilibrium temperature from holography. Phys. Rev. D 88(12), 126003 (2013). arXiv:1309.4089 [hep-th]

35. D. Giataganas, H. Soltanpanahi, Universal properties of the Langevin diffusion coefficients. Phys. Rev. D 89(2), 026011 (2014). arXiv:1310.6725 [hep-th]

36. J. Sadeghi, M.R. Setare, B. Pourhassan, Drag force with different charges in STU background and AdS/CFT. J. Phys. G 36, 115005 (2009). arXiv:0905.1466 [hep-th] 
37. M. Cvetic, Embedding AdS black holes in ten-dimensions and eleven-dimensions. Nucl. Phys. B 558, 96 (1999). arXiv:hep-th/9903214
38. J. de Boer, M. Johnstone, M.M. Sheikh-Jabbari, J. Simon, Emergent IR Dual 2d CFTs in charged AdS5 black holes. Phys. Rev. D 85, 084039 (2012). arXiv:1112.4664 [hep-th] 